\section{PREVENTION OF ANTERIOR CRUCIATE LIGAMENT INJURIES IN COMPETITIVE ALPINE SKIING}

${ }^{1}$ Maria Westin, ${ }^{1}$ Marita Harringe, ${ }^{1}$ Björn Engström, ${ }^{2}$ Marie Alricsson, ${ }^{1}$ Suzanne Werner. ${ }^{1}$ Karolinska Institutet; Sweden; ${ }^{2}$ Mid Sweden University, Sweden

\subsection{6/injuryprev-2016-042156.154}

Background Alpine skiing is one of the most popular winter sports worldwide. Like other sports alpine skiing can lead to severe injuries irrespective of skiing level. Several epidemiological studies in alpine skiers have reported the knee to be the most frequently injured body part and anterior cruciate ligament (ACL) injury the most common diagnosis. An ACL injury is serious and regardless of gender it constitutes a risk for the skier's career as well as for early osteoarthritis. Therefore, it is of utmost importance to prevent ACL injuries.

Methods This clinical trial is the fourth step in van Mechelns "Sequence of prevention" which is the optimal way to develop an injury prevention program. The intervention group consisted of all available skiers studying at a Swedish ski high school during the ski seasons 2011/2012 and 2012/2013 ( $\mathrm{n}=308$ ). Skiers, who attending the ski high schools during the seasons 2006/2007 to $2010 / 2011$ assembled to be a control group $(n=456)$. The prevention program included a video included education on awareness of how to avoid getting into ACL injury situations and exercises focusing on neuromuscular control and core stability. The goal of the prevention was to be equally good on both legs when performing different exercises indoors as well as outdoors on snow. The outcome measure consisted of the number and incidence of ACL injuries during the two year study period in comparison to the five control years.

Results In the control group 35 ACL injuries occurred showed a prevalence of $8 \%$. During the intervention years 12 ACL injuries occurred gave a prevalence of $3.9 \%$.

The absolute risk rate showed a decreased incidence rate of -0.22 (CI: $-0.44-0.00) / 100$ months attending a ski high school in favour of the intervention group.

Conclusions A prevention program focused on skiers ability to perform equilaterally can lead to prevent ACL injuries in adolescents competitive alpine skiers.

\section{RESEARCH TO SUPPORT THE IMPLEMENTATION OF A PUBLIC HEALTH POLICY ON HELMET USE IN ALPINE SKI AREAS}

Claude Goulet, ${ }^{2}$ Brent E Hagel, ${ }^{3}$ Denis Hamel, ${ }^{1,4}$ Benoit Tremblay. ${ }^{1}$ Department of Physical Education, Faculty of Education, Laval University, Québec, Canada; ${ }^{2}$ Sport Injury Prevention Research Centre, Faculty of Kinesiology and Alberta Children's Hospital Research Institute, Departments of Paediatrics and Community Health Sciences, Cumming School of Medicine, University of Calgary, Canada; ${ }^{3}$ Québec Public Health Institute, Québec, Canada; ${ }^{4}$ Safety Promotion Unit, Québec Ministry of Education, Higher Education, and Research, Canada

\subsection{6/injuryprev-2016-042156.155}

Background From 1995 to 2000, the rate of injury in Québec, Canada, ski areas significantly increased. This problem coincided with an increase in the number of snow-parks. Before suggesting injury prevention measures, some questions had to be answered: Are helmets effective? Does helmet use result in a false-sense of security? Can head-neck-helmet biomechanics increase spinal injury risk? Do expert skiers and snowboarders need a helmet? Is there an increased risk of serious injury in snow-parks? The aim of this paper is to outline how a series of epidemiologic studies significantly contributed to support the implementation of important safety measures in alpine ski areas.

Methods A series of case-control studies were undertaken. They were based on the information gathered through ski patrol injury report forms (IRF). Depending on the specific study, between 4,377 and 97,408 IRFs were analysed.

Results The results of these studies suggest that: helmets are effective in reducing the risk of any head injury (adjusted odds ratio $[\mathrm{AOR}]=0.71 ; 95 \% \mathrm{CI}: 0.55-0.92$ ); helmet use is not associated with a false-sense of security leading to riskier behaviours; helmets do not increase the risk of neck injuries; expert alpine skiers are at greater risk of head-neck injuries compared with beginners $(\mathrm{AOR}=1.86,95 \% \mathrm{CI}: 1.65-2.10)$; for skiers $(\mathrm{AOR}=1.36 ; 95 \%$ CI: 1.21-1.53) and snowboarders $(\mathrm{AOR}=1.14,95 \% \mathrm{CI}: 1.05-1.23)$, injuries sustained in snowparks compared with other slopes are more likely to require ambulance evacuation.

Conclusions Considering this evidence, the Québec Government modified a provincial regulation to make compulsory the use of helmets in all snow-parks. The Québec Ski Areas Association and the Québec Government also implemented a practical guide for building and operating snow-parks. Results show a reduction in the rates of head-neck injury from 1997-1998 season (5.07/ 10,000 visits; 95\% CI: $4.84-5.31)$ to $2012-2013$ season (3.27/ 10,000 visits; $95 \%$ CI: 3.13-3.41).

\section{THE EFFECT OF AGE ON COGNITIVE PERFORMANCE IN FINNISH SPORT-RELATED CONCUSSION ASSESSMENT}

'Kati Peltonen, 'Matti Vartiainen, ${ }^{2}$ Tiina Laitala-Leinonen, 'Laura Hokkanen'. 'Institute of Behavioural Sciences, Division of Cognitive Psychology and Neuropsychology, University of Helsinki, Finland; ${ }^{2}$ Institute of Biomedicine, Department of Cell Biology

\subsection{6/injuryprev-2016-042156.156}

Background Consensus guidelines for managing sport-related concussion in adults are being implemented in Europe, but for adolescents they are still limited. While cognitive function in adults is relatively stable over time, cognition in adolescence continues to develop. Changes in adolescents' cognitive performance have been reported to be of comparable magnitude to impairments observed on cognitive post-injury assessment in adults. Maturational improvement between baseline and post-concussion assessment may compensate any injury related cognitive impairment in adolescents, and can lead to misdiagnosis if not taken into account. The aim was to study the effect of age on cognitive performance in the Immediate Post-Concussion Assessment and Cognitive Testing (ImPACT) in Finnish adolescent ice hockey players.

Methods The ImPACT test was administered to 1311 Finnish ice hockey players at baseline for the season 2015-2016, mean age 16.31 years (standard deviation 1.79 , median 16 y, range $12-21$ y). Players were divided in seven equal sized groups (12-14, 15, $16,17,18,19$, and 20-21 years olds) and cognitive test results were compared between groups.

Results There was a statistically significant difference in cognitive test performance between age groups (Wilks' Lambda $\mathrm{p}<0.001)$. The effect appeared in all composite scores, but especially in Visual Motor Speed and Reaction Time the four first age groups showed a significant yearly change.

Conclusions Age-specific national test norms and concussion assessment guidelines are needed. Longitudinal data would enable a more reliable comparison. 